\title{
A Comparative Study on Safe Pile Capacity as Shown in Table 1 of IS 2911 (Part III): 1980
}

\author{
Somdev Pakrashi ${ }^{1}$
}

Received: 7 July 2016/Accepted: 20 April 2017/Published online: 8 May 2017

(C) The Institution of Engineers (India) 2017

\begin{abstract}
Code of practice for design and construction of under reamed pile foundations: IS 2911 (Part-III) - 1980 presents one table in respect of safe load for bored cast in situ under reamed piles in sandy and clayey soils including black cotton soils, stem dia. of pile ranging from 20 to $50 \mathrm{~cm}$ and its effective length being $3.50 \mathrm{~m}$. A comparative study, was taken up by working out safe pile capacity for one 400 dia., $3.5 \mathrm{~m}$ long bored cast in situ under reamed pile based on subsoil properties obtained from soil investigation work as well as subsoil properties of different magnitudes of clayey, sandy soils and comparing the same with the safe pile capacity shown in Table 1 of that IS Code. The study reveals that safe pile capacity computed from subsoil properties, barring a very few cases, considerably differs from that shown in the aforesaid code and looks forward for more research work and study to find out a conclusive explanation of this probable anomaly.
\end{abstract}

Keywords Under reamed pile - Safe pile capacity .

Code of practice - Comparative study

\section{Introduction}

Under-reamed piles are special type of bored piles, in general, having one or more bulbs formed by suitable enlarging of the bore-hole for the pile stem. Considerable bearing is achievable with the formation of the bulb/bulbs.

Somdev Pakrashi

s_pakrashi@rediffmail.com

1 Consultants Engineers and Builders (Geotechnical Engineering Consultancy Firm), B 294, Lake Gardens, Kolkata 700045, India
Such piles are observed to be successful and economical in sandy, clayey as well as expansive soils.

Under reamed piles are also observed to be very useful as anchors having the special advantage of resisting uplift to a great extent.

Stem diameters of these piles generally vary from 200 to $500 \mathrm{~mm}$ while effective lengths usually vary from 3.00 to 10.00 m. IS 2911, Part-III: 1980 presents a table (Table 1) containing safe load for bored cast-in-situ under-reamed piles [1].

- under vertical downward loads,

- in uplift resistance as well as

- against lateral thrust.

A study has been taken up in this paper by working out safe pile capacity (compression) of $3.50 \mathrm{~m}$ long bored cast in situ single under reamed piles having $400 \mathrm{~mm}$ stem dia., based on subsoil properties obtained from site exploration work at different sites containing sandy and clayey soils including black cotton soils as well as layered soil, in order to compare the same with the safe pile capacity (compression) shown in Table 1 of IS 2911, Part-III: 1980-for $400 \mathrm{~mm}$ stem dia. under reamed piles. This study also includes comparison of

(i) safe pile capacity worked out in respect of cohesion values from very soft to very stiff clayey soils and

(ii) sandy soils having $\Phi$ values from very loose to very dense sandy soils with the safe pile capacity (compression) shown in Table 1 of IS 2911, Part-III: 1980.

\section{Objective of the Study}

It has been observed quite a number of times by the author, as a geotechnical engineering expert, that safe pile capacity for single under reamed piles obtained from soil 
investigation work including field exploration and laboratory testing (and the safe pile capacity subsequently authenticated by initial and routine pile load tests after due technical check and approval of the safe capacity values by the client) differs considerably from the same shown in Table 1 of IS 2911 (Part-III) - 1980. This work focuses on sharing the aforesaid experience and bring about the lead for the subject of the study by presenting the evaluated safe pile capacity for a certain length and diameter of a single under reamed pile based on field/laboratory test data to compare and check if those are in conformity with the safe pile loads shown in Table 1, IS 2911, Part-III-1980 as well as examine the nature and extent of differences between the evaluated and codified safe capacity values.

\section{Assumptions}

1. To construct a perfect under ream in cohesionless soil is not practically possible. This, however, shall not affect/decrease the end bearing resistance of single under reamed piles considerably.

2. Boring operations in cohesionless soils loosen the soil and lower the end bearing resistance of single under reamed piles. Due allowances have been made for this loosening effect in evaluation for end bearing resistance from subsoil properties.

3. Evaluation of (a) safe pile capacity from subsoil properties, (b) safe pile capacity as a structural member as well as construction of under reamed piles and conducting pile load tests have all been carried out following the provisions of applicable clauses of IS 2911 (Part-III)-1980, IS 456 and other relevant IS Codes.

4. Soil Investigation and testing works at site and laboratory have all been conducted following the provisions of applicable clauses of SP 36-1 (1987), SP 36-2 (1988) and other relevant IS Codes.

5. While evaluating safe pile capacity from subsoil properties cut off level has been kept at $-1.25 \mathrm{~m}$. Minor changes in cut off level (say between -0.75 and $-2.00 \mathrm{~m}$ ) shall have no bearing on safe pile capacity obtained from sub soil properties so long as the effective length of the under reamed pile remains same i.e., $3.5 \mathrm{~m}$.

\section{Study Area and Field Studies}

Large number of soil investigation works in several parts of eastern, south and north eastern India was conducted over last four decades under the guidance of the author as a

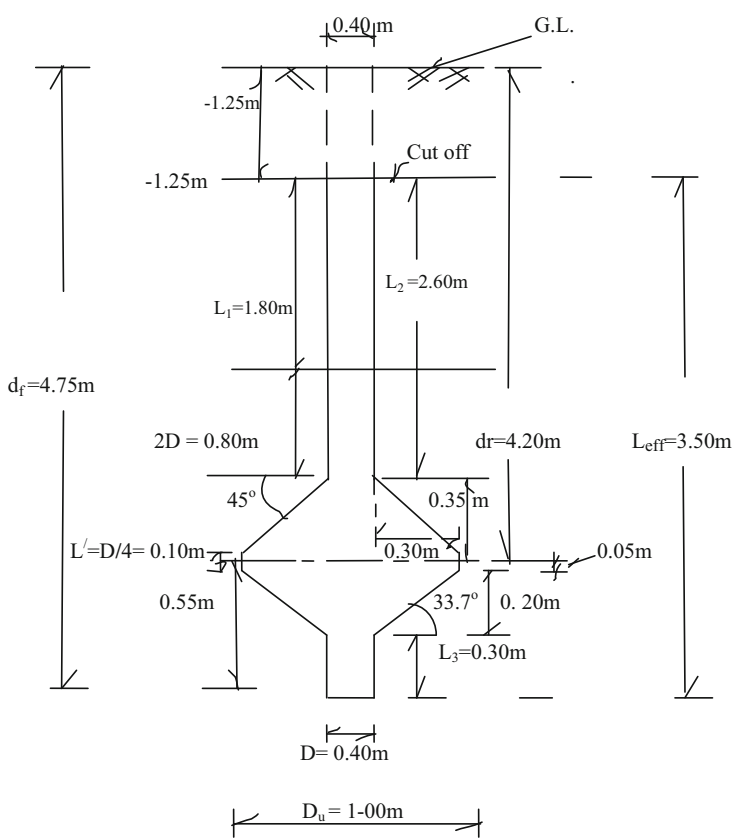

Fig. 1 A single under reamed pile with dimensions. $\mathrm{L}_{\text {eff }}=$ effective length of the under reamed pile $=3.50 \mathrm{~m}$; Cut off $=-1.25 \mathrm{~m}$; $\mathrm{L}_{1}=$ length of stem above under reamed bulb providing fictional resistance where a length of $2 \mathrm{D}$; above under ream along the stem has been ignored for friction [3] $=1.80 \mathrm{~m} ; \mathrm{L}_{2}=$ length of stem above under reamed bulb providing fictional resistance where the entire length of the stem above the under ream has been considered for friction $=2.60 \mathrm{~m} ; \mathrm{L}^{\prime}=\mathrm{D} / 4=0.10 \mathrm{~m}$, length of cylindrical part of the under reamed bulb providing fictional resistance; $\mathrm{L}_{3}=$ length of stem below under reamed bulb $=0.30 \mathrm{~m} ; \mathrm{D}_{\mathrm{f}}=$ depth of pile tip from ground level; $\mathrm{D}_{\mathrm{r}}=$ depth of centre of the cylinder of under reamed bulb; $\mathrm{D}=$ diameter of stem $=0.40 \mathrm{~m} ; \mathrm{D}_{\mathrm{u}}=$ diameter of under reamed bulb $=2.5 \times \mathrm{D}=1.00 \mathrm{~m}$

geotechnical consultant. After a thorough examination, a total of twenty-eight nos. of sites, as shown below, containing different types of subsoil deposits have been selected for this study. Safe pile capacity, for the above cases, have been presented in a tabular form to compare the same shown in Table 1 of IS 2911, Part-III: 1980.

It has also been observed by the author from his thorough experience in respect of soil investigation as well as pile load test works that safe pile capacity obtained from load tests are usually a bit higher (up to $10 \%$ at their maximum) than the same obtained from the analysis based on subsoil properties.

Besides the above, safe pile capacity in respect of very soft to very stiff clayey soils and very loose to very dense sandy subsoils have also been worked out to compare the results with the safe pile capacity shown in Table 1 of IS 2911, Part-III: 1980.

All the above exercise for the present study has been done in respect of a single under reamed pile with stem dia. $=0.40 \mathrm{~m}$, under ream dia. $=1.00 \mathrm{~m}$ and effective pile length $=3.50 \mathrm{~m}$ as shown in Fig. 1 (Tables 1, 2, 3). 
Table 1 Clayey soil 10 nos. of sites have been selected

\begin{tabular}{lll}
\hline Consistency & $\begin{array}{l}\text { No. of } \\
\text { locations }\end{array}$ & Site location and project \\
\hline Medium & 3 & $\begin{array}{l}\text { (i) Haringhata, for University Campus of West Bengal University of Technology, West Bengal } \\
\text { (ii) Haldia, for Naphtha Tankage foundation for BPCL } \\
\text { (iii) Burdwan, for P\&T Complex_Building under Postal Civil Divn. II, Yogayog Bhaban, Kolkata }\end{array}$ \\
Stiff & 4 & $\begin{array}{l}\text { (i) Bharatpur, Murshidabad, for P.O. Building \& SPM's Quarters under Postal Civil Divn, Yogayog Bhaban, } \\
\text { (ii) Polkata Panbar, Guwahati, for LIC Office Building }\end{array}$ \\
& (iii) Dispur, Assam, for Telephone Exchange Building, BSNL \\
(iv) Panbazar, Guwahati, for BSNL Admn. Building \\
(i) Royagada, Orissa, for IOCL Oil Storage Tanks \\
(ii) Balasore, Orissa, for H.P.T.V. Centre under AIR, CCW, Kolkata \\
(iii) Joranda, Orissa, for Radio Station under AIR, CCW, Kolkata
\end{tabular}

Table 2 Sandy soil 8 nos. of sites have been selected

\begin{tabular}{lll}
\hline Density & No. of locations & Site location and project \\
\hline Loose & 2 & (i) Near Jiagunge, Murshidabad, for rehabilitation of washed Railway Bridge No. 82A (E. Railway) \\
Moderately dense 6 & (ii) Lahil, Hatwar, W. Bengal, for ONGC Drill site Project \\
& (i) NIT, Durgapur, for Academic Complex \\
& (ii) Paradeep, Orissa, for BSNL S\&M building \\
& (iii) Barauni, Bihar, for Additional Tankages for IOCL \\
& (iv) Pradhan Nagar, Siliguri, for residential building for M. Design Associates, Siliguri \\
& (v) Hakimpara, Siliguri for residential building complex for M. Design Associates, Siliguri \\
& (vi) Paradeep, Orissa, for SAIL Staff Quarters
\end{tabular}

Table 3 Layered soil 10 nos. of sites have been selected

\begin{tabular}{|c|c|c|}
\hline Strength characteristics & $\begin{array}{l}\text { No. of } \\
\text { locations }\end{array}$ & Site location and project \\
\hline \multicolumn{3}{|l|}{ Clayey soil over clayey soil } \\
\hline $\begin{array}{l}\text { A. Medium clayey soil underlain by soft clayey } \\
\text { soil }\end{array}$ & 3 & $\begin{array}{l}\text { (i) Haldia Installation, BPCL, for Tankage Foundation } \\
\text { (ii) Brahmapur, Kolkata, for W.B.H. Board Residential Complex } \\
\text { (iii) Haridevpur, } 24 \text { Parganas(S), for W.B.H.B. Housing Complex }\end{array}$ \\
\hline $\begin{array}{l}\text { B. Medium clayey soil followed by very soft } \\
\text { clayey soil }\end{array}$ & 1 & (i) Dankuni Stock-Yard Complex, W. Bengal for SAIL \\
\hline \multicolumn{3}{|l|}{ Sandy soil over clayey soil } \\
\hline A. Loose sand over medium clayey soil & 3 & $\begin{array}{l}\text { (i) Bhadrak, Orissa, for Heavy duty M.W. Tower } \\
\text { under BSNL } \\
\text { (ii) KMC Compound, Garden Reach, Kolkata, for New Building with Garage } \\
\text { (iii) Eastern Rly. Colony, Liluah, Howrah, for } 0.50 \text { lac Gallon R.C.C. O.H. } \\
\text { Tank }\end{array}$ \\
\hline B. Loose sandy soil over soft clayey soil & 1 & (i) Salt Lake, Kolkata, for 8-Storied Office Building for SCERT under BSNL \\
\hline $\begin{array}{l}\text { C. Moderately dense sandy soil overlying medium } \\
\text { clayey soil }\end{array}$ & 1 & $\begin{array}{l}\text { (i) Near Govindapur Level Crossing, Kolkata in respect of Boundary Wall for } \\
\text { E. Railway }\end{array}$ \\
\hline \multicolumn{3}{|l|}{ Clayey soil over sandy soil } \\
\hline $\begin{array}{l}\text { A. Medium clayey soil followed by dense sandy } \\
\text { soil }\end{array}$ & 1 & $\begin{array}{l}\text { (i) NPT Institute, Durgapur, for Office cum Laboratory cum Auditorium } \\
\text { Building under CPWD }\end{array}$ \\
\hline
\end{tabular}


Table 4 Safe pile capacity for the under reamed pile in the sites having clayey soil—comparative study

\begin{tabular}{|c|c|c|c|c|c|c|}
\hline $\begin{array}{l}\text { Sl. } \\
\text { no. }\end{array}$ & Site location & $\begin{array}{l}\text { Client/year of } \\
\text { investigation } \\
\text { (1b) }\end{array}$ & Sub-soil profile & $\begin{array}{l}\text { Safe pile capacity as } \\
\text { per calculation based } \\
\text { on soil properties, } \\
\text { ton } \\
\text { (3) }\end{array}$ & $\begin{array}{l}\text { Safe pile capacity as } \\
\text { per Table } 1 \text {, Col. } 8 \text { of } \\
\text { IS } 2911 \text {, Part-III- } \\
\text { 1980, ton } \\
\text { (4) }\end{array}$ & $\begin{array}{l}\text { Deviation of } \\
\text { Col. (3) } \\
\text { from Col. } \\
(4), \% \\
(5)\end{array}$ \\
\hline 1. & $\begin{array}{l}\text { Haringhata for } \\
\text { Development of } \\
\text { Campus for W. Bengal } \\
\text { University of } \\
\text { Technology }\end{array}$ & $\begin{array}{l}\text { W.B. University } \\
\text { of Technology, } \\
\text { Salt Lake, } \\
\text { Kolkata/2008 }\end{array}$ & $\begin{array}{l}\text { Medium silty clay; Av. } \\
\qquad \mathrm{c}=3.8 \mathrm{t} / \mathrm{m}^{2}, \mathrm{~N}=4-5\end{array}$ & 13 & 28 & -53.57 \\
\hline 2. & $\begin{array}{l}\text { Haldia Installation for } \\
\text { Naphtha Tankage }\end{array}$ & $\begin{array}{l}\text { B.P.C.L., 15, } \\
\text { Hemanta Basu } \\
\text { Sarani, Kolkata/ } \\
2001\end{array}$ & $\begin{array}{l}\text { Medium silty clay; } \\
\quad \mathrm{c}=2.75 \mathrm{t} / \mathrm{m}^{2}, \mathrm{~N}=2-3\end{array}$ & 10.05 & 28 & -64.11 \\
\hline 3. & $\begin{array}{l}\text { Bharatpur Murshidabad } \\
\text { for P.O. Bldg. \& SPM's } \\
\text { Qtrs. for P.C. Divn.-1 }\end{array}$ & $\begin{array}{l}\text { Postal Civil Div. I, } \\
\text { Bentinck Street, } \\
\text { Kolkata/2001 }\end{array}$ & $\begin{array}{l}\text { Stiff silty clay; Av. } \\
\quad \mathrm{c}=9.85 \mathrm{t} / \mathrm{m}^{2} \\
\mathrm{~N}=9-18\end{array}$ & 32.5 & $1.25 \times 28=35$ & -7.14 \\
\hline 4. & $\begin{array}{l}\text { Burdwan P\&T Complex } \\
\text { for Div. Office } \\
\text { Building, Kolkata }\end{array}$ & $\begin{array}{l}\text { Postal Civil Div. } \\
\text { II, Bentinck } \\
\text { Street/1997 }\end{array}$ & $\begin{array}{l}\text { Medium silty clay; Av. } \\
\qquad \mathrm{c}=4.4 \mathrm{t} / \mathrm{m}^{2}, \mathrm{~N}=3-7\end{array}$ & 15.05 & 28 & -46.25 \\
\hline 5. & $\begin{array}{l}\text { Panbazar, Guwahati for } \\
\text { LIC Divn. Office }\end{array}$ & $\begin{array}{l}\text { LIC of India, } \\
\text { Guwahati, } \\
\text { Assam/1996 }\end{array}$ & $\begin{array}{l}\text { Stiff silty clay; Av. } \\
\quad \mathrm{c}=8.55 \mathrm{t} / \mathrm{m}^{2} \\
\mathrm{~N}=9-12\end{array}$ & 28.2 & $1.25 \times 28=35$ & -19.43 \\
\hline 6. & $\begin{array}{l}\text { Royagada, Orissa for } \\
\text { 2-nos. Oil Storage } \\
\text { Tanks }\end{array}$ & $\begin{array}{l}\text { IOCL, G.H. Road. } \\
\text { (S), Kolkata/ } \\
1995\end{array}$ & $\begin{array}{l}\text { Very stiff silty clay; Av. } \\
\quad \mathrm{c}=12.4 \mathrm{t} / \mathrm{m}^{2}, \text { Av. } \\
\mathrm{N}=15\end{array}$ & 39.45 & $1.25 \times 28=35$ & 12.71 \\
\hline 7. & $\begin{array}{l}\text { Balasore, Orissa for } \\
\text { H.P.TV for AIR, CCW, } \\
\text { Bhubaneswar }\end{array}$ & $\begin{array}{c}\text { All India Radio } \\
\text { Bhubaneswar } \\
\text { Orissa/1994 }\end{array}$ & $\begin{array}{l}\text { Stiff silty clay; Av. } \\
\quad \mathrm{c}=11.25 \mathrm{t} / \mathrm{m}^{2}, \\
\mathrm{~N}=13-32 ; \text { Av. c at pile } \\
\text { tip/base }=9.90 \mathrm{t} / \mathrm{m}^{2}\end{array}$ & 33.25 & $1.25 \times 28=35$ & -4.71 \\
\hline 8. & $\begin{array}{l}\text { Joranda, Orissa for Radio } \\
\text { Station }\end{array}$ & $\begin{array}{l}\text { All India Radio } \\
\text { Bhubaneswar/ } \\
1994\end{array}$ & $\begin{array}{l}\text { Stiff silty clay; Av. } \\
\qquad \mathrm{c}=10.7 \mathrm{t} / \mathrm{m}^{2} ; \mathrm{Av} \\
\mathrm{N}=30\end{array}$ & 35.3 & $1.25 \times 28=35$ & 0.86 \\
\hline 9. & $\begin{array}{l}\text { Dispur, Assam for } \\
\text { Telephone Exchange } \\
\text { Building }\end{array}$ & $\begin{array}{l}\text { Telecom Civil } \\
\text { Divn., BSNL, } \\
\text { Guwahati/1993 }\end{array}$ & $\begin{array}{l}\text { Stiff silty clay; Av. } \\
\mathrm{c}=6.25 \mathrm{t} / \mathrm{m}^{2}, \text { Av. } \\
\mathrm{N}=8-9 ; \text { Av. c at pile } \\
\text { tip/base }=6.9 \mathrm{t} / \mathrm{m}^{2}\end{array}$ & 22.5 & 28 & -19.64 \\
\hline 10. & $\begin{array}{l}\text { Panbazar, Guwahati for } \\
\text { Admn. Building for } \\
\text { BSNL }\end{array}$ & $\begin{array}{l}\text { Telecom Civil } \\
\text { Divn. BSNL } \\
\text { Guwahati/1993 }\end{array}$ & $\begin{array}{l}\text { Stiff silty clay; Av. } \\
\quad \mathrm{c}=7.5 \mathrm{t} / \mathrm{m}^{2} \\
\mathrm{~N}=10-12\end{array}$ & 24.75 & $1.25 \times 28=35$ & -29.28 \\
\hline
\end{tabular}

For this study a single under reamed pile with the dimensions [1, 2] as shown in Fig. 1 has been considered.

Comparative study, as detailed in Tables 4, 5 and 6, has been made in respect of safe capacity (compression) worked out from subsoil properties in different sandy and clayey soils including expansive black cotton soil with that shown in Table 1 of IS 2911, Part-III: 1980.

\section{Results}

Besides the above,

1. very soft to very stiff clayey soils having cohesion values ranging from 0.75 to $16 \mathrm{t} / \mathrm{m}^{2}$ as well as
2 . very loose to very dense sandy soils with ' $\Phi$ ' values ranging from $27^{\circ}$ to $45^{\circ}$ have been examined in this study in order to compare safe capacity (compression) of single under-reamed piles obtained on the basis of subsoil properties with that shown in Table 1 of IS 2911, Part-III: 1980 and presented in Tables 7 and 8 (Figs. 2, 3).

\section{Discussion and Observation}

Following observations were made in respect of the present comparative study: 
Table 5 Safe pile capacity for the under reamed pile in the sites having sandy soil—comparative study

\begin{tabular}{|c|c|c|c|c|c|c|}
\hline $\begin{array}{l}\text { S1. } \\
\text { no. }\end{array}$ & Site/location & $\begin{array}{l}\text { Client/year of } \\
\text { investigation } \\
\text { (1b) }\end{array}$ & Sub-soil profile & $\begin{array}{l}\text { Safe pile capacity } \\
\text { as per calculation } \\
\text { based on soil } \\
\text { properties, ton } \\
\text { (3) }\end{array}$ & $\begin{array}{l}\text { Safe pile capacity as } \\
\text { per Table } 1 \text { Col. } 8 \text { of } \\
\text { IS } 2911 \text {, Part-III- } \\
\text { 1980, ton } \\
\text { (4) }\end{array}$ & $\begin{array}{l}\text { Deviation } \\
\text { of Col. (3) } \\
\text { from Col. } \\
\text { (4), } \% \\
(5)\end{array}$ \\
\hline 1. & $\begin{array}{l}\text { NIT, Durgapur for } \\
\text { Academic Complex }\end{array}$ & $\begin{array}{l}\text { Ex. Engr.(C), CPWD, } \\
\text { KCD-VI, Nizam } \\
\text { Palace, AJC Bose } \\
\text { Rd., Kolkata/2007 }\end{array}$ & $\begin{array}{l}\text { Moderately dense sand; Av. } \\
\quad \Phi=31^{\circ}, \mathrm{N}=6-18\end{array}$ & 25.4 & 28 & -9.29 \\
\hline 2. & $\begin{array}{l}\text { Near Jiagunge, } \\
\text { Murshidabad for } \\
\text { rehabilitation of } \\
\text { Washed Bridge No. } \\
\text { 82A (E. Rly.) }\end{array}$ & $\begin{array}{l}\text { M/s. L.K. Industries } \\
\text { 21/48, Karaya Road, } \\
\text { Kolkata/2001 }\end{array}$ & $\begin{array}{l}\text { Loose silty fine to medium } \\
\text { sand; Av. } \Phi=29.5^{\circ} \text {, Av. } \\
\mathrm{N}=8-9\end{array}$ & 21.35 & $28 \times 0.75=21$ & 1.67 \\
\hline 3. & $\begin{array}{l}\text { Paradeep, Orissa for } \\
\text { S\&M Building for } \\
\text { BSNL }\end{array}$ & $\begin{array}{l}\text { Telecom Civil Divn., } \\
\text { Friends Colony, Nala } \\
\text { Road, Cuttack/1999 }\end{array}$ & $\begin{array}{l}\text { Moderately dense fine sand; } \\
\text { Av. } \Phi=32^{\circ} \text {, Av. } \\
\mathrm{N}=16\end{array}$ & 29.4 & 28 & 5.00 \\
\hline 4. & $\begin{array}{l}\text { Barauni, Bihar for prop. } \\
\text { 3-nos. Additional } \\
\text { Tankage for IOCL }\end{array}$ & $\begin{array}{l}\text { I.O.C.L., 2, G.H. Road } \\
\text { (S) Kolkata/1997 }\end{array}$ & $\begin{array}{l}\text { Moderately dense silty fine } \\
\text { sand; Av. } \Phi=35^{\circ} \\
\mathrm{N}=11-16\end{array}$ & 47.4 & 28 & 69.29 \\
\hline 5. & $\begin{array}{l}\text { Pradhan Nagar, Siliguri } \\
\text { for residential } \\
\text { Building }\end{array}$ & $\begin{array}{l}\text { Modern Design } \\
\text { Associates 149/97, } \\
\text { Hill Cart Road, } \\
\text { Pradhan Nagar, } \\
\text { Siliguri/1996 }\end{array}$ & $\begin{array}{l}\text { Moderately dense silty fine } \\
\text { sand; Av. } \Phi=31^{\circ} .5 \text { Av. } \\
\mathrm{N}=13\end{array}$ & 27.35 & 28 & -2.32 \\
\hline 6. & $\begin{array}{l}\text { Hakimpara, Siliguri for } \\
\text { residential Building }\end{array}$ & $\begin{array}{l}\text { Modern Design } \\
\text { Associates, Sevoke } \\
\text { Road, Siliguri/1996 }\end{array}$ & $\begin{array}{l}\text { Moderately dense silty fine } \\
\text { sand; Av. } \Phi=30.5^{\circ} \\
\mathrm{N}=14-15 ; \text { At pile tip/ } \\
\text { base: } \Phi=31^{\circ}\end{array}$ & 25.35 & 28 & -9.46 \\
\hline 7. & $\begin{array}{l}\text { Paradeep, Orissa for } \\
\text { SAII Staff Quarters }\end{array}$ & $\begin{array}{l}\text { SAIL. Rabindra Sarani, } \\
\text { Kolkata/1990 }\end{array}$ & $\begin{array}{l}\text { Moderately dense fine to } \\
\text { medium sand; Av. } \\
\Phi=33^{\circ}, \text { Av. } N=15 ; \text { At } \\
\text { pile tip/base: } \Phi=34^{\circ}\end{array}$ & 38.3 & 28 & 36.79 \\
\hline 8. & $\begin{array}{l}\text { Lahil Hatwar, W. Bengal } \\
\text { Drill site for ONGC } \\
\text { Project }\end{array}$ & $\begin{array}{l}\text { ONGC, W.B. P-45, } \\
\text { Taratala Road, } \\
\text { Kolkata/1989 }\end{array}$ & $\begin{array}{l}\text { Loose fine to medium sand; } \\
\text { Av. } \Phi=29^{\circ} .5 \text {, Av. } \\
N=9\end{array}$ & 21.35 & $28 \times 0.75=21$ & 1.67 \\
\hline
\end{tabular}

\section{When the Under Ream Pile is in Clayey Soil}

(a) For very soft to soft clayey soil $\left(\mathrm{c} \leq 3.00 \mathrm{t} / \mathrm{m}^{2}\right)$ [3, 4], safe pile capacity values from subsoil properties are observed to be quite smaller in comparison to safe loads as per the IS Code, its deviation from safe loads as per IS 2911 (Part-III) - 1980 varying between -80.37 and $-47.86 \%$.

(b) For medium clayey soil (where cohesion, $c>3.00 \mathrm{t} /$ $\mathrm{m}^{2}$ but $\leq 6.00 \mathrm{t} / \mathrm{m}^{2}$ ) [3, 4], safe pile capacity obtained from subsoil properties is also much lower, its deviation from safe loads as per IS 2911 (PartIII) - 1980 varying between -60.36 and $-26.79 \%$.

(c) For a range from 6 to $9 \mathrm{t} / \mathrm{m}^{2}$ of stiff clayey soil [3, 4], safe pile capacity as obtained from subsoil properties has also been observed to be considerably lower, its deviation from safe load as per the above IS Code varying between -41.09 and $-15.14 \%$. (d) Only for the next range of stiff to very stiff clayey soil $\left[3\right.$, 4], having $\mathrm{c} \geq 9.50 \mathrm{t} / \mathrm{m}^{2}$ but $\leq 12.50 \mathrm{t} / \mathrm{m}^{2}$, safe pile capacity obtained from subsoil properties is very close to the safe pile capacity as per the said IS Code, its deviation from the safe pile load as shown in the above IS Code varying between -10.43 and $13.71 \%$.

(e) For very stiff to hard clayey soils [3, 4] having c $>12.50 \mathrm{t} / \mathrm{m}^{2}$ but $\leq 16.00 \mathrm{t} / \mathrm{m}^{2}$, safe pile capacity value as obtained from subsoil properties is more, in each case, than the safe pile capacity as per IS Code. Here deviation of safe pile capacity obtained from subsoil properties vary between 18.14 and $45.43 \%$, from the safe pile load as per the above IS Code.

(f) It is also observed that the safe pile capacity obtained on the basis of subsoil properties keeps on increasing with the increment of cohesion value. At $c=0.75 \mathrm{t} /$ $\mathrm{m}^{2}$, safe pile capacity calculated on subsoil properties works out to 2.75 ton and for $\mathrm{c}=16 \mathrm{t} / \mathrm{m}^{2}$, safe pile 
Table 6 Safe pile capacity for the under reamed pile in the sites having layered soil—comparative study

\begin{tabular}{lll}
\hline Sl. Site/location & Client/year of & Sub-soil profile \\
No & investigation &
\end{tabular}

(1) (1a)

1. N.P.T. Institute, Durgapur for Office cum Lab. cum Auditorium Bldg.

2. Bhadrak, Orissa for Heavy Duty Microwave Tower

(1b)

Ex. Engr.(C), CPWD, First layer: up to $-3.05 \mathrm{~m}$ KCD-VI, Nizam Palace, AJC Bose Road, Kolkata/2010

Medium silty clay with some coarse sand; $c=4.25 \mathrm{t} / \mathrm{m}^{2}$, $\mathrm{N}=10$

Second layer: below $-3.05 \mathrm{~m}$

Dense coarse sand; $\Phi=38.5^{\circ}$

First layer: up to $-2.25 \mathrm{~m}$

Loose fine to medium sand;

Av. $\Phi=29^{\circ}$, Av. $\mathrm{N}=5$

Second layer: below $-2.25 \mathrm{~m}$

Medium to stiff silty clay; Av. $\mathrm{c}=4.95 \mathrm{t} / \mathrm{m}^{2}$, Av. $\mathrm{N}=7-8$;

Av. c at pile tip/base $=5.75 \mathrm{t} /$ $\mathrm{m}^{2}, \mathrm{~N}=8-11$

3. Near Govindapur Level Crossing, Kolkata for Boundary Wall

4. Haldia Installation BPCL for 4-nos. Tankage

Foundation

5. Salt Lake, Kolkata for PWD (C.Brd.), West 8-Storied. Office Building for SCERT Bengal, Kolkata/ 1998

BPCL, West Bengal, Kolkata/1998

1998

First layer: up to $-3 \mathrm{~m}$

Moderately dense silty fine sand; Av. $\Phi=31^{\circ}$, Av.

$\mathrm{N}=7$

Second Layer: below $-3 \mathrm{~m}$

Medium silty clay; Av.

$\mathrm{c}=3.1 \mathrm{t} / \mathrm{m}^{2}$, Av. $\mathrm{N}=4-6$

First layer: up to $-3.6 \mathrm{~m}$

Medium silty clay; Av.

$$
\mathrm{c}=2.85 \mathrm{t} / \mathrm{m}^{2}, \mathrm{~N}=3-5
$$

Second layer: from $-3.6 \mathrm{~m}$

Soft silty clay with decayed and decomposed vegetation; Av. $\mathrm{c}=1.85 \mathrm{t} / \mathrm{m}^{2} \mathrm{~N}=1-3$

First layer: up to $-3 \mathrm{~m}$

Loose silty sand; Av. $\Phi=29^{\circ}$, $\mathrm{N}=2-5$

Next layer: from $-3 \mathrm{~m}$

Soft silty clay; Av. $\mathrm{c}=1.95 \mathrm{t} /$ $\mathrm{m}^{2}$, Av. $\mathrm{N}=2-3$

6. Garden Reach, CMC Calcutta Municipal compound for New Corporation S.N. Building with Garage provision

7. Brahmapur, Kolkata for Residential Complex
28

Safe pile capacity Safe pile capacity [1] Deviation as per calculation as per Table $1 \mathrm{Col} .8$ of Col. (3) based on soil of IS 2911, Part-III- from Col. properties, ton 1980 , ton $\quad$ (4), \%

(3) (4)

(5)

88.2

$1.25 \times 28=35$
Banerjee Road,

Kolkata/1998

West Bengal Housing Board Kolkata/1997

First layer: up to $-3.5 \mathrm{~m}$

Loose silty fine sand; Av.

$\Phi=29^{\circ}, \mathrm{N}=5$

Next layer: from $-3.5 \mathrm{~m}$

Soft silty clay; Av. c $=3 \mathrm{t} / \mathrm{m}^{2}$, Av. $\mathrm{N}=3-4$

First layer: up to $-4 \mathrm{~m}$

Medium silty clay; Av. $\mathrm{c}=3.70 \mathrm{t} / \mathrm{m}^{2}, \mathrm{~N}=3-8$

Second layer: below $-4 \mathrm{~m}$

Soft silty clay with decayed and decomposed vegetation; Av. $\mathrm{c}=1.75 \mathrm{t} / \mathrm{m}^{2} ; \mathrm{N}=1-3$ 
Table 6 continued

\begin{tabular}{|c|c|c|c|c|c|c|}
\hline $\begin{array}{l}\text { Sl. } \\
\text { No }\end{array}$ & Site/location & $\begin{array}{l}\text { Client/year of } \\
\text { investigation } \\
\text { (1b) }\end{array}$ & $\begin{array}{l}\text { Sub-soil profile } \\
\text { (2) }\end{array}$ & $\begin{array}{l}\text { Safe pile capacity } \\
\text { as per calculation } \\
\text { based on soil } \\
\text { properties, ton } \\
\text { (3) }\end{array}$ & $\begin{array}{l}\text { Safe pile capacity [1] } \\
\text { as per Table } 1 \text { Col. } 8 \\
\text { of IS } 2911 \text {, Part-III- } \\
1980 \text {, ton } \\
\text { (4) }\end{array}$ & $\begin{array}{l}\text { Deviation } \\
\text { of Col. (3) } \\
\text { from Col. } \\
(4), \% \\
(5)\end{array}$ \\
\hline 8. & $\begin{array}{l}\text { Liluah E. Rly. Colony } \\
\text { Howrah for } 0.50 \\
\text { Lac gallon RCC } \\
\text { O.H. Tank }\end{array}$ & $\begin{array}{l}\text { Eastern Rly. Strand } \\
\text { Road, Howrah/1997 }\end{array}$ & $\begin{array}{l}\text { First layer: up to }-3.35 \mathrm{~m} \\
\text { Loose silty fine sand; Av. } \\
\Phi=30^{\circ} ; \text { Av. } \mathrm{N}=3 \\
\text { Second layer: from }-3.35 \mathrm{~m} \\
\text { Medium silty clay with } \\
\text { decomposed vegetation; Av. } \\
\mathrm{c}=2.25 \mathrm{t} / \mathrm{m}^{2}\end{array}$ & 7.6 & $0.75 \times 28=21$ & -63.81 \\
\hline 9. & $\begin{array}{l}\text { Haridevpur, } 24 \\
\text { Pgs.(S) for Housing } \\
\text { Complex }\end{array}$ & $\begin{array}{l}\text { W.B. Housing Board, } \\
\text { 105, S.N. Banerjee } \\
\text { Road, Kolkata/1995 }\end{array}$ & $\begin{array}{l}\text { First layer: up to }-3.35 \mathrm{~m} \\
\text { Soft/medium silty clay; Av. } \\
\mathrm{c}=2.45 \mathrm{t} / \mathrm{m}^{2}, \mathrm{~N}=2-3 \\
\text { Next layer: below }-3.35 \mathrm{~m} \\
\text { Soft silty clay with decayed and } \\
\text { decomposed vegetation; Av. } \\
\mathrm{c}=2.01 \mathrm{t} / \mathrm{m}^{2}, \mathrm{~N}=0-1\end{array}$ & 7.6 & $0.75 \times 28=21$ & -63.81 \\
\hline 10. & $\begin{array}{l}\text { Dankuni Stockyard } \\
\text { Complex, W. } \\
\text { Bengal, for SAIL }\end{array}$ & $\begin{array}{l}\text { SAIL, Rabindra Sarani, } \\
\text { Kolkata/1989 }\end{array}$ & $\begin{array}{l}\text { First layer: up to }-1.75 \mathrm{~m} \\
\text { Medium silty clay; Av. } \mathrm{c}=4 \mathrm{t} / \\
\mathrm{m}^{2}, \mathrm{~N}=5-6 \\
\text { Second layer: below }-1.75 \mathrm{~m} \\
\text { Very soft silty clay with } \\
\text { decayed and decomposed } \\
\text { vegetation; Av. } \mathrm{c}=0.9 \mathrm{t} / \mathrm{m}^{2} \text {, } \\
\text { Av. } \mathrm{N}=1-2\end{array}$ & 3.65 & $0.5 \times 28=14$ & -73.93 \\
\hline
\end{tabular}

Table 7 Comparative study for safe pile capacity: under reamed pile in clayey soil

\begin{tabular}{|c|c|c|c|c|c|}
\hline $\begin{array}{l}\text { Consistency of sub } \\
\text { soil }[3,4]\end{array}$ & $\begin{array}{l}\text { Sl. } \\
\text { no. } \\
\text { (1) }\end{array}$ & $\begin{array}{l}\mathrm{c} \\
\mathrm{t} / \mathrm{m}^{2} \\
(2)\end{array}$ & $\begin{array}{l}\text { Safe pile capacity as per calculation based } \\
\text { on soil properties, ton } \\
\text { (3) }\end{array}$ & $\begin{array}{l}\text { Safe pile capacity [1] as per Table } 1, \text { Col-8 of IS } \\
2911 \text { (Part-III): 1980, ton } \\
\text { (4) }\end{array}$ & $\begin{array}{l}\text { Deviation of Col. (3) } \\
\text { from Col. (4), \% } \\
\text { (5) }\end{array}$ \\
\hline \multirow[t]{4}{*}{ Very Soft } & 1. & 0.75 & 2.75 & $0.5 \times 28=14$ & -80.37 \\
\hline & 2. & 1 & 3.65 & $0.5 \times 28=14$ & -73.93 \\
\hline & 3. & 1.25 & 4.55 & $0.5 \times 28=14$ & -67.50 \\
\hline & 4. & 1.5 & 5.5 & $0.5 \times 28=14$ & -60.71 \\
\hline \multirow[t]{5}{*}{ Soft } & 1. & 1.75 & 6.4 & $0.75 \times 28=21$ & -69.52 \\
\hline & 2. & 2 & 7.3 & $0.75 \times 28=21$ & -65.24 \\
\hline & 3. & 2.25 & 8.25 & $0.75 \times 28=21$ & -60.71 \\
\hline & 4. & 2.5 & 9.15 & $0.75 \times 28=21$ & -56.43 \\
\hline & 5. & 3 & 10.95 & $0.75 \times 28=21$ & -47.86 \\
\hline \multirow[t]{12}{*}{ Medium } & 1. & 3.25 & 11.1 & 28 & -60.36 \\
\hline & 2. & 3.5 & 11.95 & 28 & -57.32 \\
\hline & 3. & 3.75 & 12.8 & 28 & -54.29 \\
\hline & 4. & 4 & 13.65 & 28 & -51.25 \\
\hline & 5. & 4.25 & 14.5 & 28 & -48.21 \\
\hline & 6. & 4.5 & 15.4 & 28 & -45.00 \\
\hline & 7. & 4.75 & 16.25 & 28 & -41.96 \\
\hline & 8. & 5 & 17.1 & 28 & -38.93 \\
\hline & 9. & 5.25 & 17.95 & 28 & -35.89 \\
\hline & 10. & 5.5 & 18.8 & 28 & -32.85 \\
\hline & 11. & 5.75 & 19.65 & 28 & -29.82 \\
\hline & 12. & 6 & 20.5 & 28 & -26.79 \\
\hline
\end{tabular}


Table 7 continued

\begin{tabular}{|c|c|c|c|c|c|}
\hline $\begin{array}{l}\text { Consistency of sub } \\
\text { soil }[3,4]\end{array}$ & $\begin{array}{l}\text { Sl. } \\
\text { no. } \\
(1)\end{array}$ & $\begin{array}{l}\mathrm{c}, \\
\mathrm{t} / \mathrm{m}^{2} \\
(2)\end{array}$ & $\begin{array}{l}\text { Safe pile capacity as per calculation based } \\
\text { on soil properties, ton } \\
\text { (3) }\end{array}$ & $\begin{array}{l}\text { Safe pile capacity [1] as per Table } 1, \text { Col- } 8 \text { of IS } \\
2911 \text { (Part-III): } 1980 \text {, ton } \\
\text { (4) }\end{array}$ & $\begin{array}{l}\text { Deviation of Col. (3) } \\
\text { from Col. (4), \% } \\
\text { (5) }\end{array}$ \\
\hline \multirow[t]{13}{*}{ Stiff } & 1. & 6.25 & 20.65 & $1.25 \times 28=35$ & -41.09 \\
\hline & 2. & 6.5 & 21.45 & $1.25 \times 28=35$ & -38.71 \\
\hline & 3. & 7 & 23.1 & $1.25 \times 28=35$ & -34.00 \\
\hline & 4. & 7.5 & 24.75 & $1.25 \times 28=35$ & -29.29 \\
\hline & 5. & 8 & 26.4 & $1.25 \times 28=35$ & -24.57 \\
\hline & 6. & 8.5 & 28.05 & $1.25 \times 28=35$ & -19.86 \\
\hline & 7. & 9 & 29.7 & $1.25 \times 28=35$ & -15.14 \\
\hline & 8. & 9.5 & 31.35 & $1.25 \times 28=35$ & -10.43 \\
\hline & 9. & 10 & 33 & $1.25 \times 28=35$ & -5.71 \\
\hline & 10. & 10.5 & 34.65 & $1.25 \times 28=35$ & -1.00 \\
\hline & 11. & 11 & 36.3 & $1.25 \times 28=35$ & 3.71 \\
\hline & 12. & 11.5 & 37.95 & $1.25 \times 28=35$ & 8.43 \\
\hline & 13. & 12 & 39.6 & $1.25 \times 28=35$ & 13.14 \\
\hline \multirow[t]{9}{*}{ Very stiff to hard } & 1 & 12.25 & 39 & $1.25 \times 28=35$ & 11.43 \\
\hline & 2 & 12.5 & 39.8 & $1.25 \times 28=35$ & 13.71 \\
\hline & 3 & 13 & 41.35 & $1.25 \times 28=35$ & 18.14 \\
\hline & 4 & 13.5 & 42.95 & $1.25 \times 28=35$ & 22.71 \\
\hline & 5 & 14 & 44.55 & $1.25 \times 28=35$ & 27.29 \\
\hline & 6 & 14.5 & 46.15 & $1.25 \times 28=35$ & 31.86 \\
\hline & 7 & 15 & 47.75 & $1.25 \times 28=35$ & 36.43 \\
\hline & 8 & 15.5 & 49.3 & $1.25 \times 28=35$ & 40.86 \\
\hline & 9 & 16 & 50.9 & $1.25 \times 28=35$ & 45.43 \\
\hline
\end{tabular}

Table 8 Comparative study for safe pile capacity: under reamed pile in sandy soil

\begin{tabular}{|c|c|c|c|c|c|}
\hline $\begin{array}{l}\text { Density of sub } \\
\text { soil }[3,5]\end{array}$ & $\begin{array}{l}\text { S1. } \\
\text { no. } \\
(1)\end{array}$ & $\begin{array}{l}\Phi \\
\text { deg } \\
(2)\end{array}$ & $\begin{array}{l}\text { Safe pile capacity as per calculation } \\
\text { based on soil properties, ton } \\
\text { (3) }\end{array}$ & $\begin{array}{l}\text { Safe pile capacity [1] as per Table } 1 \text { Col- } 8 \text { of } \\
\text { IS } 2911 \text { Part-III: } 1980 \text {, ton } \\
\text { (4) }\end{array}$ & $\begin{array}{l}\text { Deviation of Col. ( } 3 \text { ) } \\
\text { from Col. (4), \% } \\
(5)\end{array}$ \\
\hline \multirow[t]{4}{*}{ Very loose } & 1 & 27 & 14.15 & $0.50 \times 28=14$ & 1.07 \\
\hline & 2 & 27.5 & 16.2 & $0.50 \times 28=14$ & 15.71 \\
\hline & 3 & 28 & 17.2 & $0.50 \times 28=14$ & 22.86 \\
\hline & 4 & 28.5 & 18.7 & $0.50 \times 28=14$ & 33.57 \\
\hline \multirow[t]{3}{*}{ Loose } & 1 & 29 & 20.15 & $0.75 \times 28=21$ & -4.05 \\
\hline & 2 & 29.5 & 21.35 & $0.75 \times 28=21$ & 1.67 \\
\hline & 3 & 30 & 22.75 & $0.75 \times 28=21$ & 8.33 \\
\hline \multirow{12}{*}{$\begin{array}{l}\text { Moderately } \\
\text { dense }\end{array}$} & 1 & 30.5 & 24.5 & 28 & -12.50 \\
\hline & 2 & 31 & 25.4 & 28 & -9.29 \\
\hline & 3 & 31.5 & 27.35 & 28 & -2.32 \\
\hline & 4 & 32 & 29.4 & 28 & 5.00 \\
\hline & 5 & 32.5 & 32.25 & 28 & 15.18 \\
\hline & 6 & 33 & 34 & 28 & 21.43 \\
\hline & 7 & 33.5 & 36.4 & 28 & 30.00 \\
\hline & 8 & 34 & 38.35 & 28 & 36.96 \\
\hline & 9 & 34.5 & 41.8 & 28 & 49.29 \\
\hline & 10 & 35 & 47.4 & 28 & 69.29 \\
\hline & 11 & 35.5 & 52.1 & 28 & 86.07 \\
\hline & 12 & 36 & 56.7 & 28 & 102.50 \\
\hline
\end{tabular}


Table 8 continued

\begin{tabular}{|c|c|c|c|c|c|}
\hline $\begin{array}{l}\text { Density of sub } \\
\text { soil }[3,5]\end{array}$ & $\begin{array}{l}\text { Sl. } \\
\text { no. } \\
(1)\end{array}$ & $\begin{array}{l}\Phi \\
\text { deg } \\
(2)\end{array}$ & $\begin{array}{l}\text { Safe pile capacity as per calculation } \\
\text { based on soil properties, ton } \\
\text { (3) }\end{array}$ & $\begin{array}{l}\text { Safe pile capacity [1] as per Table } 1 \text { Col- } 8 \text { of } \\
\text { IS } 2911 \text { Part-III: } 1980 \text {, ton } \\
\text { (4) }\end{array}$ & $\begin{array}{l}\text { Deviation of Col. (3) } \\
\text { from Col. (4), } \% \\
\text { (5) }\end{array}$ \\
\hline \multirow[t]{10}{*}{ Dense } & 1 & 36.5 & 63.05 & $1.25 \times 28=35$ & 80.14 \\
\hline & 2 & 37 & 67.2 & $1.25 \times 28=35$ & 92.00 \\
\hline & 3 & 37.5 & $75.65^{\mathrm{a}}$ & $1.25 \times 28=35$ & 116.14 \\
\hline & 4 & 38 & $80.05^{\mathrm{a}}$ & $1.25 \times 28=35$ & 128.71 \\
\hline & 5 & 38.5 & $89.95^{\mathrm{a}}$ & $1.25 \times 28=35$ & 157.00 \\
\hline & 6 & 39 & $101.1^{\mathrm{a}}$ & $1.25 \times 28=35$ & 188.86 \\
\hline & 7 & 39.5 & $106.4^{\mathrm{a}}$ & $1.25 \times 28=35$ & 204.00 \\
\hline & 8 & 40 & $121.15^{\mathrm{a}}$ & $1.25 \times 28=35$ & 246.14 \\
\hline & 9 & 40.5 & $130.8^{\mathrm{a}}$ & $1.25 \times 28=35$ & 273.71 \\
\hline & 10 & 41 & $147.65^{\mathrm{a}}$ & $1.25 \times 28=35$ & 321.86 \\
\hline \multirow[t]{8}{*}{ Very dense } & 1 & 41.5 & $165.65^{\mathrm{a}}$ & $1.25 \times 28=35$ & 373.29 \\
\hline & 2 & 42 & $180.2^{\mathrm{a}}$ & $1.25 \times 28=35$ & 414.86 \\
\hline & 3 & 42.5 & $186.3^{\mathrm{a}}$ & $1.25 \times 28=35$ & 432.29 \\
\hline & 4 & 43 & $192.35^{\mathrm{a}}$ & $1.25 \times 28=35$ & 449.57 \\
\hline & 5 & 43.5 & $210.4^{\mathrm{a}}$ & $1.25 \times 28=35$ & 501.14 \\
\hline & 6 & 44 & $228.5^{\mathrm{a}}$ & $1.25 \times 28=35$ & 552.86 \\
\hline & 7 & 44.5 & $268.9^{\mathrm{a}}$ & $1.25 \times 28=35$ & 668.29 \\
\hline & 8 & 45 & $309.3^{\mathrm{a}}$ & $1.25 \times 28=35$ & 783.71 \\
\hline
\end{tabular}

${ }^{a}$ Structural safe capacity for the under reamed pile with M25 R.C.C. $=75$ ton

capacity is observed to be 18.50 times high as it works out to 50.90 ton. But the safe pile capacity as per the said IS Code varies between a smaller range of 14-35 ton and the ratio between the maximum and the minimum value is 2.5 only.

\section{When the Under Ream Pile is in Sandy Soil}

1. For very loose/loose sandy soil $\left(27^{\circ} \leq \Phi \leq 30^{\circ}\right)[3]$

(a) Safe pile capacity as obtained from subsoil properties is observed to be more than that as per the above IS Code where, $\Phi$ values $\geq 27.5^{\circ}$ but $\leq 28.5^{\circ}$. Here deviation of safe pile capacity obtained from subsoil properties vary between 15.71 and $33.57 \%$, from the safe pile load as per the above IS Code.

(b) Safe pile capacity obtained from subsoil properties and the same as per the said IS Code is almost equal or very close to each other for $\Phi=27^{\circ}$ as well as where $29^{\circ} \leq \Phi \leq 30^{\circ}$, i.e., for a very small range. The deviation of safe pile capacity based on subsoil properties from that shown in the aforesaid IS Code are $1.07 \%$ and -4.05 to $8.33 \%$, deviations being negligible or very small.
2. For moderately dense sandy soil $\left(30^{\circ}<\Phi \leq 36^{\circ}\right)[3]$

(a) Safe pile capacity obtained from subsoil properties and the same as per the said IS Code are very close to each other (\% deviation varies from -9.29 to 5 ) where $31^{\circ} \leq \Phi \leq 32^{\circ}$.

(b) Safe pile capacity values obtained from subsoil properties are somewhat higher $(\%$ deviation varies from 15.18 to 30.00 ) than those shown in the above mentioned IS Code-for $\phi$ values $\geq 32.5^{\circ}$ but $\leq 33.5^{\circ}$, whereas, for $\phi=30.5^{\circ}$, safe capacity obtained from subsoil properties is bit less $(\%$ deviation $=-12.5)$ than that shown in the said IS Code.

(c) Safe pile capacity obtained from subsoil properties, having $\phi$ values $>33.5^{\circ}$ but $\leq 36^{\circ}$, however, become considerably higher $(\%$ deviation $=36.96-102.5)$ than those as per the said IS Code.

3. For dense to very dense sandy soil $\left(36.5^{\circ} \leq \Phi \leq 45^{\circ}\right)$ [3]

Percentage deviations of the safe pile capacity obtained from subsoil properties are observed to be very large varying between 80.14 and 783.71 .

4. For sandy soil it is also observed that the safe pile capacity obtained on the basis of subsoil properties 
$\mathbf{a}$

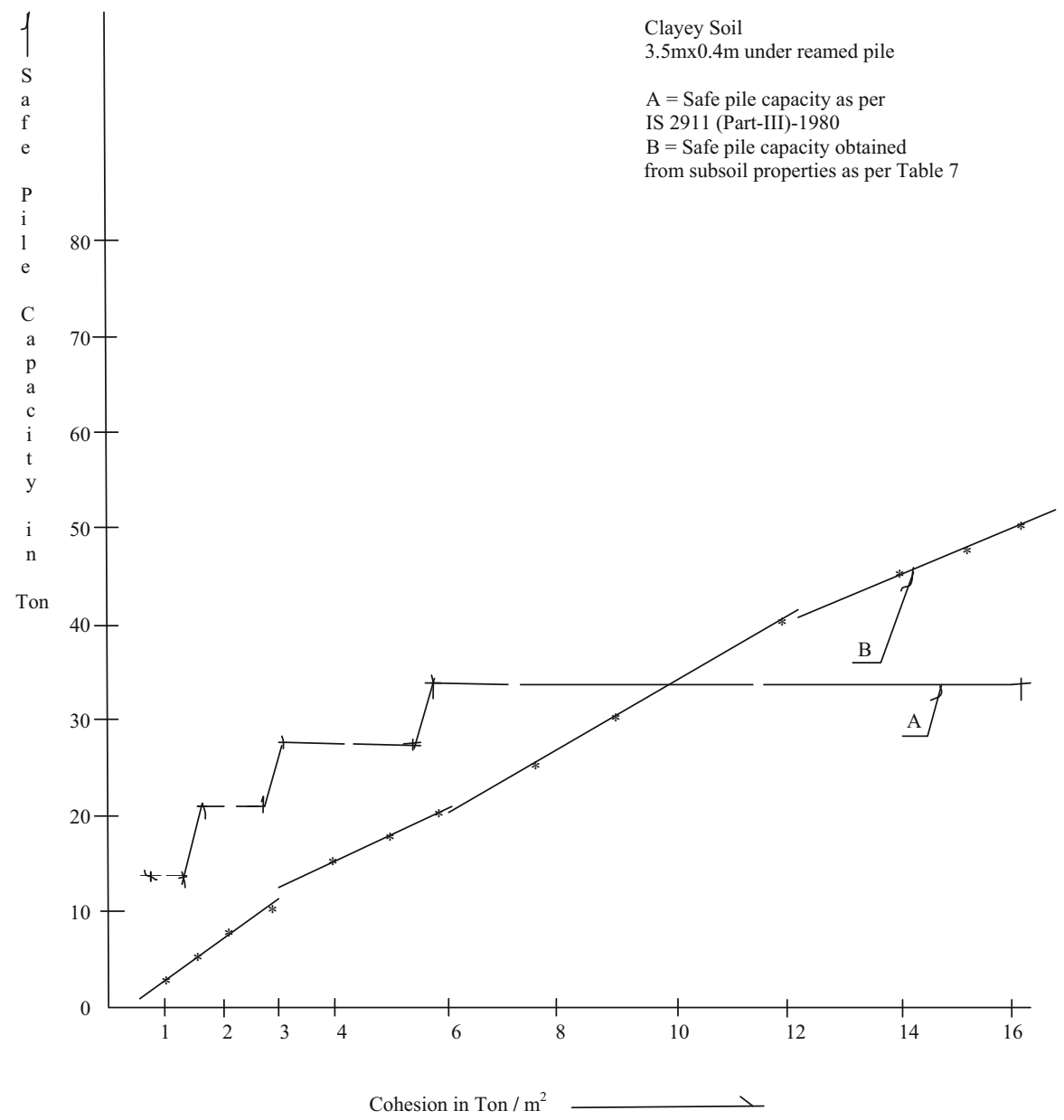

Fig. 2 a Safe pile capacity versus cohesion curves. b Safe pile capacity versus angle of internal friction curves

keeps on increasing with the increment of ' $\Phi$ ' value. At $\Phi=27^{\circ}$, safe pile capacity calculated from subsoil properties works out to 14.15 ton and for $\Phi=45^{\circ}$, worked out safe pile capacity is 21.86 times of 14.15 ton, being 309.30 ton. It has, however, been observed that structural safe pile capacity (compression) for a $3.5 \mathrm{~m}$ long $\times 400 \mathrm{~mm}$ stem dia. under reamed pile with M20 and M25 R.C.C are 65 and 75 ton respectively. Accordingly these structural safe pile capacity values, respectively, are 4.59 and 5.3 times higher than 14.15 ton. But safe pile capacity as per IS Code, in comparison, varies between quite a smaller range of 14 and 35 ton for the same range of ' $\Phi$ ' values, i.e., between $27^{\circ}$ and $45^{\circ}$. The ratio between the highest and the lowest value of safe pile load is 2.5 only.

\section{When the Under Ream Pile is in Layered Soil}

Safe pile capacity obtained from subsoil properties, as well, increases with the increment of ' $c$ ' and/or ' $\Phi$ ' values of the subsoil whereas the safe pile capacity given in the IS Code is same for a wide range of strength parameters and variation range of the same is quite small being from 14 to 35 ton only.

Deviation (\%) of Safe Pile Capacity Based on Subsoil Properties from the Safe Pile Load Shown in the IS 2911, Part-III: 1980

(a) In case of clayey soils, aforesaid deviations are negative (from -80.37 to $-1.00 \%$ ) as well as diminishing with the increment of 'c' values, from 0.75 to 
Sandy Soil

b

$3.5 \mathrm{mx} 0.4 \mathrm{~m}$ under reamed pile

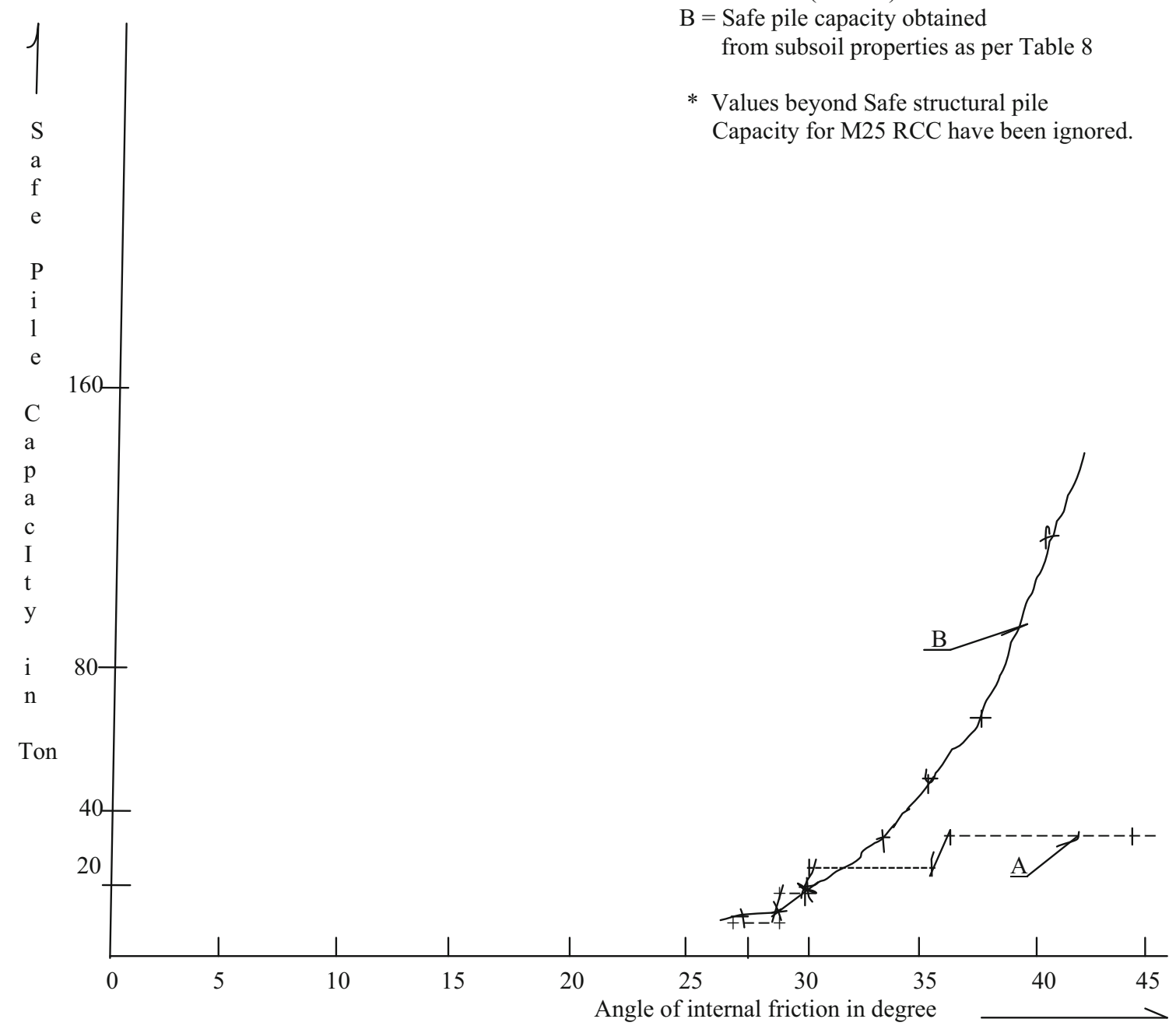

Fig. 2 continued

$10.5 \mathrm{t} / \mathrm{m}^{2}$. With the increment of cohesion values from 11 to $16 \mathrm{t} / \mathrm{m}^{2}$ said deviations go on increasing (3.71-45.43\%) all values being positive.

(b) In case of sandy soils, deviations (from $1.07 \%$ at ' $\Phi$ ' $=27^{\circ}$ to $783.71 \%$ at ' $\Phi$ ' $=45^{\circ}$ ) are mostly positive and go on increasing with the increment of ' $\Phi$ '. However the deviations are negative only for ' $\Phi$ ' $=29^{\circ}, 30.5^{\circ}, 31^{\circ}$ and $31.5^{\circ}$, the deviations being $-4.05,-12.5,-9.29$ and $-2.32 \%$ respectively.

(c) For layered soil, Table 6, shows large deviation of values based on subsoil properties from those as per the IS Code and the values are mostly negative.

\section{Limitations}

(a) Influence of field and laboratory test procedures, some conditions and faults by manpower in carrying out soil investigation work etc. is not unlikely on study results.

(b) This study has been carried out in respect of one particular length and diameter of one single under reamed pile. Quite a number of study/research work in respect of under reamed piles having different pile lengths and diameters in various sites with different 
Fig. 3 a Deviation (\%) of safe pile capacity based on subsoil properties from that shown in IS 2911, Part-III-1980.

b Deviation (\%) of safe pile capacity based on subsoil properties from that shown in IS 2911, Part-III-1980 $\mathbf{a}$

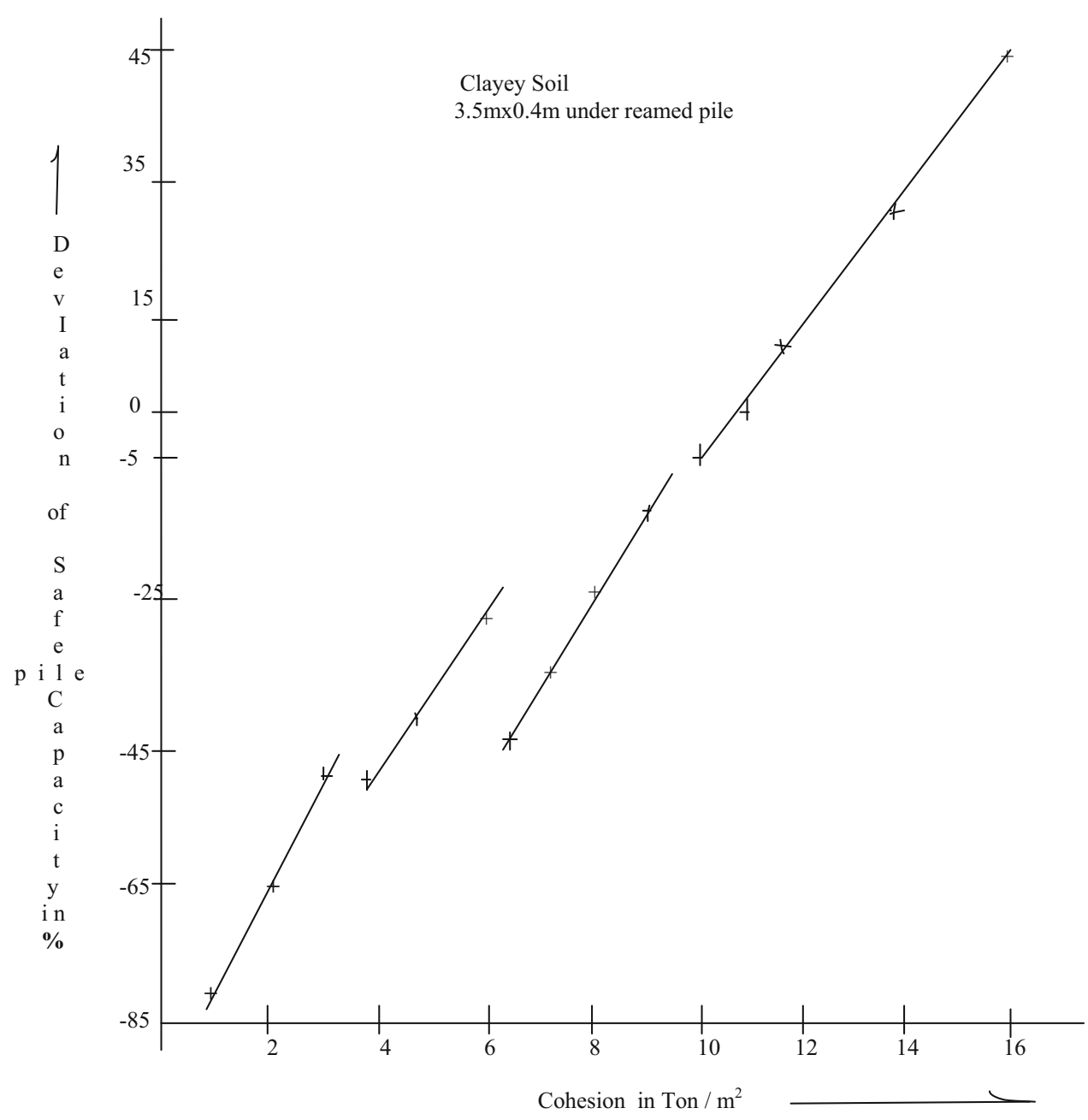

sub soil compositions/conditions etc. are necessary before any conclusive explanation/decision can be made in respect of this comparative study.

\section{Conclusions}

A comparative study on safe loads (compression) as shown in Table 1 of IS 2911 Part-III-1980 was taken up by working out safe pile capacity (compression) from soil properties of different subsurface deposits in respect of $3.5 \mathrm{~m}$ long bored cast in situ single under ream piles having $400 \mathrm{~mm}$ stem dia.- to examine whether the results are in conformity with the safe loads (compression) shown in the said table of the said IS Code.

Study results show that the safe pile capacity as obtained from subsoil properties differs, from the safe pile capacity shown in the said table of the said IS Code, in most of the cases to a great extent in sandy and clayey soils including black cotton soil as well as in layered soil i.e., in all types of subsoils, except for the small range of values where $c \geq 9.5 \mathrm{t} / \mathrm{m}^{2}$ but $\leq 12.5 \mathrm{t} / \mathrm{m}^{2}$ as well as $\Phi=27^{\circ}, \Phi \geq 29^{\circ}$ but $\leq 30^{\circ}$ and $\Phi \geq 31^{\circ}$ but $\leq 32^{\circ}$.

Safe pile capacity obtained on the basis of subsoil properties keeps on increasing with the increment of ' $c$ ' and/or ' $\Phi$ ' values for all types of subsoils including layered soil and the increment is manyfold from low $\left(\mathrm{c}=0.75 \mathrm{t} / \mathrm{m}^{2}\right.$ or $\left.\Phi=27^{\circ}\right)$ to high $\left(\mathrm{c}=16 \mathrm{t} / \mathrm{m}^{2}\right.$ or $\left.\Phi=45^{\circ}\right)$ shear strength parameters. Ratio between maximum and minimum safe pile capacity for clayey soil is 18.5 and for sandy soil it is 21.76 . Safe capacity in terms of structural safety of this pile with M20 and M25 RCC are however 65 and 75 ton respectively. With a safe pile capacity value $=75$ ton, aforesaid ratio of maximum to minimum safe pile capacity for sandy soil is to 5.30. But, safe pile capacity given in the said IS Code, for all types of subsoil including black cotton soil and layered soil is same for a wide range of strength parameters and its variation 
b

Sandy Soil

$3.5 \mathrm{mx} 0.4 \mathrm{~m}$ under reamed pile

* Deviation for values beyond Safe structural

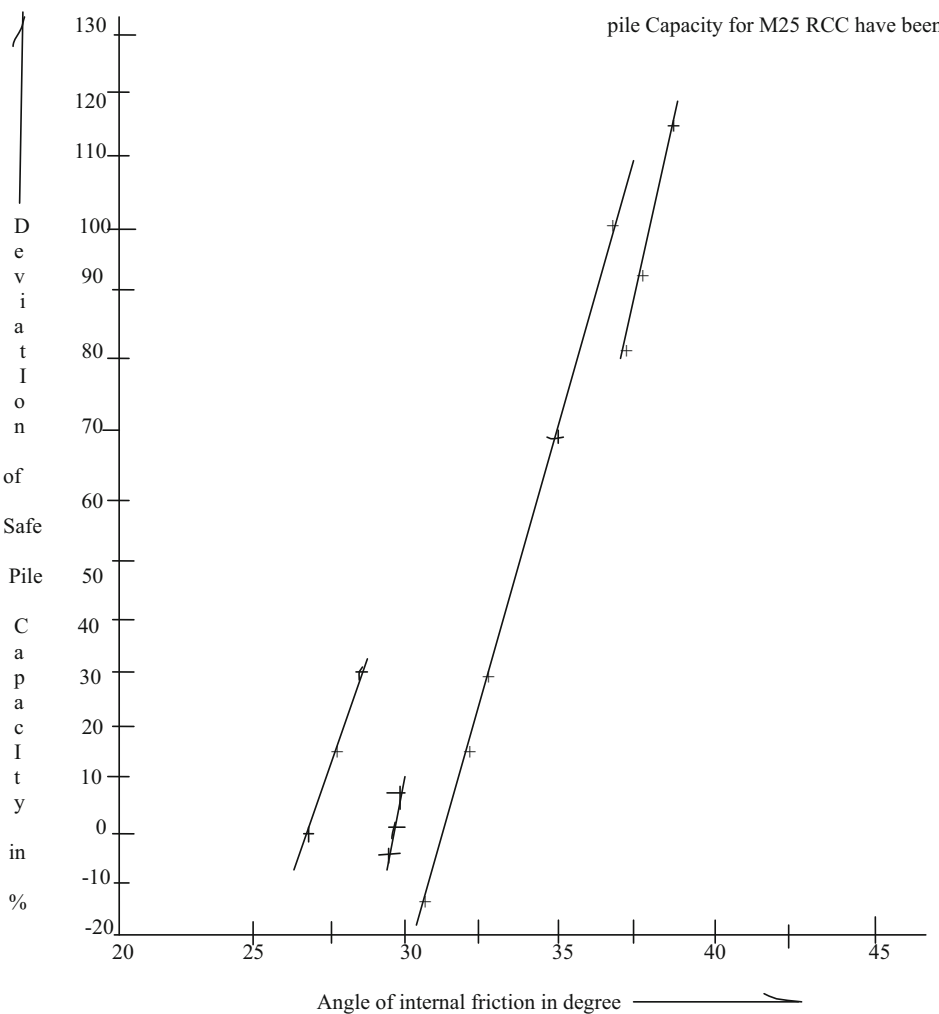

Fig. 3 continued

range is also quite small (14-35 ton), the ratio between the values being as low as 2.5 .

It is observed that some civil engineers refer to the aforesaid table of IS 2911, Part-III, 1980 as a ready means of obtaining safe load for under reamed piles in sandy/clayey soil including black cotton soil or layered subsoil i.e., for all types of subsoils. Under the light of the above findings and observations it appears, therefore, prudent to look forward to more study and research work in order to find out a conclusive explanation in regard to probable anomaly of the results arising out from the present study.

\section{Appendix 1: Expression used for the determination of safe pile capacity for single under reamed pile} [1]

\section{For Clayey Soils}

$$
\begin{aligned}
\mathrm{Q}_{\mathrm{a}}= & \mathrm{Q}_{\mathrm{d} 1} \mathrm{~F} \\
= & \left(\mathrm{A}_{\mathrm{p}} \mathrm{N}_{\mathrm{c}} \mathrm{c}_{\mathrm{p}}+\mathrm{A}_{\mathrm{a}} \mathrm{N}_{\mathrm{c}} \mathrm{c}_{\mathrm{a}}^{\prime}+\mathrm{c}_{\mathrm{s} 1}^{\prime} A_{\mathrm{s} 1}^{\prime} \alpha_{1}^{\prime}+\mathrm{c}_{\mathrm{s} 2}^{\prime} \mathrm{A}_{\mathrm{s} 2}^{\prime} \alpha_{2}\right. \\
& \left.+\mathrm{c}_{\mathrm{s} 3}^{\prime} \mathrm{A}_{\mathrm{s} 3}^{\prime} \alpha_{3}\right) / \mathrm{F}
\end{aligned}
$$

where $Q_{a}\left(t / m^{2}\right)=$ safe bearing capacity of pile; $F=$ factor of safety $=2.5$ (taken); $\mathrm{Q}_{\mathrm{d}}\left(\mathrm{t} / \mathrm{m}^{2}\right)=$ ultimate bearing capacity of pile; $A_{p}=\pi / 4 D^{2}$, cross-sectional area of pile stem at toe level; $\mathrm{N}_{\mathrm{c}}=$ bearing capacity factor, taken as 9 ; $c_{p}=$ cohesion of soil around toe; $A_{a}=\pi / 4\left(D_{u}^{2}-D^{2}\right)$ where $D_{u}$ and $D$ are the under-reamed bulb and stem diameters respectively; $\mathrm{c}_{\mathrm{a}}^{\prime}=$ average cohesion of soil around under reamed bulb; $\alpha_{1}, \alpha_{2}, \alpha_{3}=$ reduction factors; $\mathrm{c}^{\prime} \mathrm{s}_{1}=$ average cohesion of soil along pile stem above under reamed bulb; $\mathrm{A}^{\prime} \mathrm{s}_{1}=$ surface area of the stem above under reamed bulb providing frictional resistance; $\mathrm{c}^{\prime} \mathrm{s}_{2}=$ cohesion of soil around the under-reamed bulb; $\mathrm{A}^{\prime} \mathrm{s}_{2}=\pi \mathrm{D}_{\mathrm{u}}(\mathrm{D} / 4)$, surface area of the cylinder circumscribing the under reamed bulb; $c^{\prime} s_{3}=$ average cohesion of soil along pile stem below the under reamed bulb; and $\mathrm{A}^{\prime} \mathrm{s}_{3}=$ surface area of stem below the under reamed bulb.

(I) First two terms of the above expression are for bearing and the last three are for friction components.

(II) For uplift first term of expression (i) will not occur.

(III) Frictional resistance for a length of $2 \mathrm{D}$ along the stem above the under reamed bulb has not been considered owing to disturbance of that part of 
subsoil due to possible settlement of the under reamed bulb.

(IV) Where $c_{p}=c^{\prime}$ a first two terms reduces to

$$
\left(\mathrm{Ap}+\mathrm{A}_{\mathrm{a}}\right) \mathrm{Nc} \times \mathrm{c}^{\prime} \mathrm{a}=\pi / 4 \mathrm{Du}^{2} \mathrm{Nc} \times \mathrm{c}^{\prime} \mathrm{a}
$$

(V) Where $\mathrm{c}_{\mathrm{p}}=\mathrm{c}^{\prime} \mathrm{a}=\mathrm{c}_{\mathrm{s} 1}^{\prime}=\mathrm{c}_{\mathrm{s} 2}^{\prime}=\mathrm{c}_{\mathrm{s} 3}^{\prime}=\mathrm{c}=$ cohesion of soil between -1.25 and $-4.75 \mathrm{~m}$, we have, $\alpha_{1}=\alpha_{2}=\alpha_{3}=\alpha$ and expression (i) becomes

$\mathrm{Q}_{\mathrm{a}}=\mathrm{Q}_{\mathrm{d}} / \mathrm{F}=\left\{(\pi / 4) \mathrm{D}_{\mathrm{u}}^{2} \mathrm{~N}_{\mathrm{C}} \mathrm{c}+\mathrm{c} \alpha\left(\mathrm{A}_{\mathrm{s} 1}^{\prime}+\mathrm{A}_{\mathrm{s} 2}^{\prime}+\mathrm{A}_{\mathrm{s} 3}^{\prime}\right)\right\} / \mathrm{F}$

From Fig. 1 we have, $L_{\text {eff. }}=3.50 \mathrm{~m} ; \mathrm{D}=0.40 \mathrm{~m}$; $\mathrm{D}_{\mathrm{u}}=2.5 \times 0.40=1.00 \mathrm{~m} ; \mathrm{L}_{1}=1.80 \mathrm{~m} ; \mathrm{L}_{2}=2.60 \mathrm{~m} ;$ $\mathrm{L}_{3}=0.3 \mathrm{~m} ; \mathrm{L}^{\prime}=\mathrm{D} / 4=0.10 \mathrm{~m}$.

$\mathrm{F}=2.5, \therefore$ from expression (ib) we have, $\mathrm{Q}_{\mathrm{a}}=\mathrm{Q}_{\mathrm{d}} /$ $\mathrm{F}=\{(\pi /$

4) $\left.\times(1)^{2} \times \mathrm{c} \times 9+\pi \times 0.4 \times \mathrm{c} \alpha(1.80+0.25+0.3)\right\} /$ $\mathrm{F}=(7.0686+2.9531 \alpha) \mathrm{c} / \mathrm{F}$.

For $\alpha=0.7\left(\mathrm{c} \leq 3 \mathrm{t} / \mathrm{m}^{2}\right)$ [6], Qa $=3.654 \mathrm{c}$; for $\alpha=0.5$ (c $>3 \mathrm{t} / \mathrm{m}^{2}$ but $\left.\leq 6.0 \mathrm{t} / \mathrm{m}^{2}\right)$ [6], Qa $=3.418 \mathrm{c}$; for $\alpha=0.4$ (c $>6.0 \mathrm{t} / \mathrm{m}^{2}$ but $\left.\leq 12.0 \mathrm{t} / \mathrm{m}^{2}\right)$ [6], Qa $=3.300 \mathrm{c}$; for $\alpha=0.3\left(\mathrm{c}>12.0 \mathrm{t} / \mathrm{m}^{2}\right)[6], \mathrm{Qa}=3.182 \mathrm{c}$.

\section{For Sandy Soils}

$$
\begin{aligned}
\mathrm{Q}_{\mathrm{a}}= & \mathrm{Q}_{\mathrm{d}} / \mathrm{F}=\left\{\mathrm{Ap}\left\{1 / 2 \gamma \mathrm{DN} \gamma+\gamma \mathrm{d}_{\mathrm{f}} \mathrm{Nq}\right\}\right. \\
& +\mathrm{A}_{\mathrm{a}}\left\{1 / 2 \gamma \mathrm{D}_{\mathrm{u}} \mathrm{N} \gamma+\gamma \mathrm{d}_{\mathrm{r}} \mathrm{N}_{\mathrm{q}}\right) \\
& +\mathrm{A}^{\prime} \mathrm{s}_{1} \mathrm{~K} \tan \delta \mathrm{pd}_{1}+\mathrm{A}^{\prime} \mathrm{s}_{2} \mathrm{~K} \tan \delta \mathrm{p}_{\mathrm{d} 2} \\
& \left.+\mathrm{A}^{\prime} \mathrm{s}_{3} \mathrm{~K} \tan \delta \mathrm{pd}_{3}\right\} / \mathrm{F}
\end{aligned}
$$

where $\mathrm{Q}_{\mathrm{a}}\left(\mathrm{t} / \mathrm{m}^{2}\right)=$ safe bearing capacity of pile; $\mathrm{F}=$ factor of safety $=2.5$ (taken); $\mathrm{Q}_{\mathrm{d}}\left(\mathrm{t} / \mathrm{m}^{2}\right)=$ ultimate bearing capacity of pile; $A_{p}=\pi / 4 D^{2} ; A_{a}=\pi / 4\left(D_{u}^{2}-D^{2}\right) ; p_{d 1}=$ average effective over burden pressure along stem above under reamed bulb; $\mathrm{p}_{\mathrm{d} 2}=$ average effective overburden pressure at the cylinder circumscribing the under reamed bulb; $\mathrm{p}_{\mathrm{d} 3}=$ average effective over burden pressure at stem below under reamed bulb; $\mathrm{A}^{\prime} \mathrm{s}_{1}=$ surface area of the stem above under reamed bulb providing frictional resistance; $\mathrm{A}^{\prime} \mathrm{s}_{2}=\pi \mathrm{D}_{\mathrm{u}} \cdot(\mathrm{D} /$ 4), surface area of the cylinder circumscribing the under reamed bulb; $\mathrm{A}^{\prime} \mathrm{s}_{3}=$ surface area of stem below the under reamed bulb; $\gamma=$ average effective unit weight of soil; $\mathrm{N} \gamma$ and $\mathrm{N}_{\mathrm{q}}=$ bearing capacity factors depending upon the angle of internal friction; $\mathrm{d}_{\mathrm{f}}=$ total depth of pile below ground level; $\mathrm{d}_{\mathrm{r}}=$ depth of the centre of under-reamed bulb below ground level; $\mathrm{K}=$ earth pressure coefficient; $\delta=$ angle of wall friction (taken equal to $0.85 \times$ angle of internal friction Ф) $[3,5] ; \mathrm{L}_{1}=$ length of stem above under reamed bulb providing fictional resistance where a length $=2 \mathrm{D}$ above the under reamed bulb [3] has not been considered for frictional resistance $=1.80 \mathrm{~m} ; \mathrm{L}_{2}=$ entire length of the stem above under reamed bulb $=2.60 \mathrm{~m} ; \mathrm{L}_{3}=$ length of stem below under reamed bulb $=0.30 \mathrm{~m}$.

(1) First two terms of the above expression are for bearing and the last three are for friction components

(2) For uplift, bearing on tip, $A_{p}$ will not occur. Where $\Phi$ is same throughout the subsoil from cut off to tip of the pile expression (ii) becomes

$$
\begin{aligned}
\mathrm{Q}_{\mathrm{a}}= & \mathrm{Q}_{\mathrm{d}} / \mathrm{F}=\left\{\mathrm{Ap}\left(1 / 2 \gamma \mathrm{DN} \gamma+\gamma \mathrm{d}_{\mathrm{f}} \mathrm{Nq}\right)\right. \\
& +\mathrm{A}_{\mathrm{a}}\left(1 / 2 \gamma \mathrm{D}_{\mathrm{u}} \mathrm{N} \gamma+\gamma \mathrm{d}_{\mathrm{r}} \mathrm{N}_{\mathrm{q}}\right) \\
& \left.+\mathrm{K} \tan \delta\left(\mathrm{A}^{\prime} \mathrm{s}_{1} \mathrm{pd}_{1}+\mathrm{A}^{\prime} \mathrm{s}_{2} \mathrm{p}_{\mathrm{d} 2}+\mathrm{A}^{\prime} \mathrm{s}_{3} \mathrm{pd}_{3}\right)\right\} / \mathrm{F}
\end{aligned}
$$

For very loose to moderately dense sandy soils $\left(\Phi \geq 27^{\circ}\right.$ but $\left.\leq 36^{\circ}\right)[3,7]$, frictional resistance along the stem for a length of $2 \mathrm{D}$ above the under ream has not been considered.

Now, with $\gamma=0.85, \mathrm{~L}_{1}=1.80 \mathrm{~m}, \mathrm{~L}_{3}=0.30 \mathrm{~m}$, $\mathrm{D}=0.40 \mathrm{~m}, \mathrm{Du}=1.00 \mathrm{~m}, \mathrm{~d}_{\mathrm{f}}=4.75 \mathrm{~m}$ and $\mathrm{d}_{\mathrm{r}}=4.20 \mathrm{~m}$, the above expression becomes,

$$
\begin{aligned}
\mathrm{Q}_{\mathrm{a}}= & \mathrm{Q}_{\mathrm{d}} / \mathrm{F}=[(\pi / 4) \times 0.16(0.5 \times 0.85 \times 0.4 \times \mathrm{N} \gamma \\
& +0.85 \times 4.75 \times \mathrm{Nq}) \\
& +(\pi / 4) \times 0.84(0.5 \times 0.85 \times 1 \times \mathrm{N} \gamma \\
& \left.+0.85 \times 4.2 \times \mathrm{N}_{\mathrm{q}}\right) \\
& +\pi \times 0.4 \times \mathrm{K} \tan \delta \mathrm{c} 0.85 \\
& \{1.80 \times 2.15+(1 / 4) \times 4.2+0.3 \times 4.60\}] / \mathrm{F} \\
= & {[0.02136 \mathrm{~N} \gamma+0.50737 \mathrm{Nq}+0.28039 \mathrm{~N} \gamma} \\
& +2.3553 \mathrm{~N}_{\mathrm{q}} \\
& +\pi \times 0.4 \times \mathrm{K} \tan \delta \times 0.85 \\
& \times\{1.80 \times 2.15+(1 / 4) \times 4.2 \\
& +0.3 \times 4.60\}] / 2.5 \\
= & \left(0.30175 \mathrm{~N} \gamma+2.86267 \mathrm{~N}_{\mathrm{q}}+6.7293 \mathrm{~K} \tan \delta\right) \\
& / 2.5=0.1207 \mathrm{~N} \gamma+1.1451 \mathrm{~N}_{\mathrm{q}} \\
& +2.6917 \mathrm{~K} \tan \delta
\end{aligned}
$$

For dense to very dense sandy soils $\left(\Phi>36^{\circ}\right)[3,7]$ entire length above the under ream is considered to provide frictional resistance.

Here, with $\gamma=0.85, \mathrm{~L}_{1}=2.60 \mathrm{~m}, \quad \mathrm{~L}_{3}=0.30 \mathrm{~m}$ $\mathrm{D}=0.40 \mathrm{~m}, \mathrm{Du}=1.00 \mathrm{~m}, \mathrm{~d}_{\mathrm{f}}=4.75 \mathrm{~m}$ and $\mathrm{d}_{\mathrm{r}}=4.20 \mathrm{~m}$ expression (iia) reduces to

$$
\begin{aligned}
\mathrm{Q}_{\mathrm{a}}= & \mathrm{Q}_{\mathrm{d}} / \mathrm{F}=[\pi / 4 \times 0.16 \times(1 / 2 \times 0.85 \times 0.4 \mathrm{~N} \gamma \\
& +0.85 \times 4.75 \mathrm{Nq}) \\
& +\pi / 4 \times 0.84 \times\left(1 / 2 \times 0.85 \mathrm{~N} \gamma+0.85 \times 4.2 \times \mathrm{N}_{\mathrm{q}}\right) \\
& +\pi \times 0.4 \times \mathrm{K} \tan \delta 0.85 \\
& \times\{2.60 \times 2.55+(1 / 4) \times 4.2+0.3 \times 4.60\}] / \mathrm{F} \\
= & \left(0.30175 \mathrm{~N} \gamma+2.86237 \mathrm{~N}_{\mathrm{q}}+9.6774 \mathrm{~K} \tan \delta\right) / 2.5 \\
= & 0.1207 \mathrm{~N} \gamma+1.1451 \mathrm{~N}_{\mathrm{q}}+3.871 \mathrm{~K} \tan \delta
\end{aligned}
$$


Taken, $\mathrm{K}=1$ for $\Phi \leq 30^{\circ} ; \mathrm{K}=1.25$ for $\Phi>30^{\circ}$ but $\leq 32^{\circ} ; \mathrm{K}=1.50$ for $\Phi>32^{\circ}$ but $\leq 36^{\circ}$; $\mathrm{K}=1.75$ for $\Phi>36^{\circ}$ but $\leq 38^{\circ}$; K $=2.00$ for $\Phi>38^{\circ}$ [3].

\section{Appendix 2: Values of $N_{q}$ and $N \gamma$ as per relevant IS Code $\left(\Phi^{\prime}=\Phi-2\right.$, considering loosening of subsoil at pile tip)}

\begin{tabular}{llllll}
\hline$\Phi^{\prime}$ & $\mathrm{N}_{\mathrm{q}}[1]$ & $\mathrm{N} \gamma[7]$ & $\Phi^{\prime}$ & $\mathrm{N}_{\mathrm{q}}[1]$ & $\mathrm{N} \gamma[7]$ \\
\hline 25 & 10.20 & 10.88 & 35 & 50 & 48.03 \\
25.5 & 11.85 & 12.032 & 35.5 & 56.7 & 54.168 \\
26 & 12.60 & 13.184 & 36 & 59.8 & 60.306 \\
26.5 & 13.75 & 14.336 & 36.5 & 67.2 & 66.444 \\
27 & 14.9 & 15.488 & 37 & 76.2 & 72.582 \\
27.5 & 15.8 & 16.64 & 37.5 & 80.1 & 78.72 \\
28 & 16.85 & 17.792 & 38 & 92.3 & 84.858 \\
28.5 & 17.95 & 18.944 & 38.5 & 100 & 90.996 \\
29 & 18.6 & 20.096 & 39 & 114 & 97.13 \\
29.5 & 20.15 & 21.218 & 39.5 & 129 & 103.27 \\
30 & 21.8 & 22.40 & 40 & 141 & 109.41 \\
30.5 & 23.7 & 24.963 & 40.5 & 144.5 & 125.645 \\
31 & 24.9 & 27.526 & 41 & 148 & 141.88 \\
31.5 & 26.7 & 30.089 & 41.5 & 162 & 158.115 \\
32.0 & 28.10 & 32.652 & 42 & 176 & 174.35 \\
32.5 & 30.8 & 35.215 & 42.5 & 209.5 & 190.585 \\
33 & 35.4 & 37.778 & 43 & 243 & 206.82 \\
33.5 & 39.2 & 40.341 & 43.5 & 271 & 223.055 \\
34 & 42.9 & 42.904 & 44 & 299 & 239.29 \\
34.5 & 46.7 & 45.467 & 45 & 345 & 271.76 \\
\hline & & & & &
\end{tabular}

\section{Appendix 3: Sample Calculation}

\section{Determination of safe pile capacity (compression) from necessary subsoil parameters for the single under reamed pile}

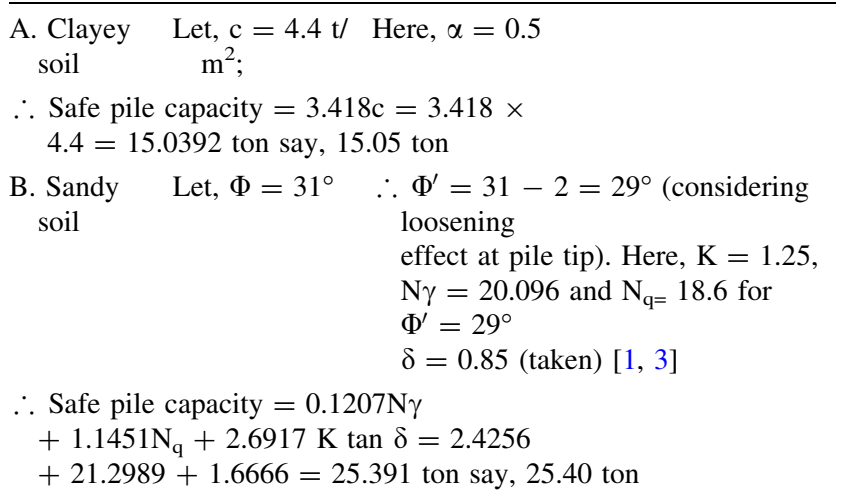

Estimate of safe capacity in respect of structural safely of the single under reamed pile

\begin{tabular}{ll}
\hline Safe capacity (compression) & Use M20 RCC [8] \\
$=1372.79 \times 51 \times 10^{-3}$ & $\mathrm{f}_{\mathrm{sc}}=51 \mathrm{~kg} / \mathrm{cm}^{2} ; \mathrm{m}=13.33$ \\
$=70.01$ ton & $\Phi=40 \mathrm{~cm} ;$ \\
$\therefore$ Use safe capacity $=65$ ton & $\mathrm{A}_{\mathrm{s}}=\mathrm{A}_{\mathrm{g}}+(\mathrm{m}-1) \times \mathrm{A}_{\mathrm{s}}$ \\
Now, $65 \times 10^{3} / 1372.79$ & $=(\pi / 4) \times 40^{2}+12.33 \times 9.42$ \\
$=47.35 \mathrm{~kg} / \mathrm{cm}^{2}<51$ ton $\therefore$ & $=1372.79 \mathrm{~cm}^{2}$ \\
O.K. & Used 3 nos. $16 \mathrm{~mm} \Phi$ \\
& and 3 nos. $12 \mathrm{~mm}^{2} \Phi$, \\
& area $=09.42 \mathrm{~cm}^{2}>$ min. steel \\
& $4) \times(40)^{2}=5.03 \mathrm{~cm}^{2} \therefore$ O.K. \\
Safe capacity (compression) & Use M25 RCC [8] \\
$=1350.65 \times 10^{-3} \times 61.2=$ & $\mathrm{f}_{\mathrm{sc}}=61.2 \mathrm{~N} \mathrm{~kg} / \mathrm{cm}^{2} ; \mathrm{m}=10.98$ \\
82.66 ton & $\Phi=40 \mathrm{~cm} ;$ \\
$\therefore$ Use safe capacity $=75$ ton & $\mathrm{A}_{\mathrm{s}}=\mathrm{A}_{\mathrm{g}}+(\mathrm{m}-1) \mathrm{A}_{\mathrm{s}}$ \\
Now, $75 \times 10^{3} /$ & $=(\pi / 4) \times 40^{2}+9.98 \times$ \\
$1350.65=55.53 \mathrm{~kg} /$ & $9.42=1350.65 \mathrm{~cm}^{2}$ \\
cm $<61.2$ ton $\therefore$ O.K. & Use, 3 nos. $16 \mathrm{~mm} \Phi+$ \\
& 3 nos. $12 \Phi$, area $=09.42 \mathrm{~cm}^{2}$ \\
& $>$ min. steel@0.4\% of $(\pi /$ \\
& $4) \times 40^{2}=5.03 \mathrm{~cm}^{2}$ \\
& $\therefore$ O.K. \\
\hline
\end{tabular}

\section{References}

1. IS: 2911 (Part-III) - 1980, Indian Standard Code of Practice for Design and Construction of Pile Foundations, Part-III, UnderReamed Piles (First Revision). Indian Standard Institution, Manak Bhavan, 9, Bahadur Shah Zafar Marg, New Delhi 110002, February (1981)

2. SP 7(2005), National Building Code Of India 2005, Second Reprint 2007, Bureau of Indian. Standards, Manak Bhavan, New Delhi 110002, Part 6, Section 2, Fig. 7, p. 30

3. M.J. Tomlinson, Foundation Design and Construction, 4th edn. The English Language Book Society and Pitman, ELBS edition reprinted (1981)

4. K. Terzaghi, R.B. Peck, Soil Mechanics in Engineering Practice, First Indian Edition, Eleventh Printing, August 1960 (Asia Publishing House, Calcutta, 1962)

5. R.B. Peck, W.E. Hanson, T.H. Thornburn, Foundation Engineering,2nd. Edition,John Wiley and Sons, New York, USA, 1974

6. IS: 2911 (Part I/Sec 2) - 2010, Indian Standard Code of Practice for Design and Construction of Pile Foundations, Part I: Concrete Piles, Section 2: Bored Cast In Situ Concrete Piles (Second Revision). Bureau of Indian Standards, Manak Bhavan, 9, Bahadur Shah Zafar Marg, New Delhi 110002, May 2011, p. 25

7. IS: 6403 - 1981, Indian Standard Code of Practice for Determination of Bearing Capacity of Shallow Foundations. Indian Standard Institution, Manak Bhavan, 9, Bahadur Shah Zafar Marg, New Delhi 110002, November 1981

8. IS 456-2000, Indian Standard Plain and Reinforced ConcreteCode of Practice (Fourth Revision) Bureau of Indian Standards, Manak Bhavan, 9 Bahadur Shah Zafar Marg, New Delhi 110002, July 2000 